\title{
BISM \\ ONLINE \\ One-year follow-up of a randomised controlled trial on added splinting to eccentric exercises in chronic midportion Achilles tendinopathy
}

Suzan de Jonge, Robert-Jan de Vos, Hans T.M. van Schie, Jan A.N. Verhaar, Adam Weir and Johannes L. Tol

Br. J. Sports Med. published online 6 Oct 2008;

doi:10.1136/bjsm.2008.052142

Updated information and services can be found at:

http://bjsm.bmj.com/cgi/content/abstract/bjsm.2008.052142v1

\section{These include:}

Rapid responses You can respond to this article at:

http://bjsm.bmj.com/cgi/eletter-submit/bjsm.2008.052142v1

Email alerting Receive free email alerts when new articles cite this article - sign up in the box at the service top right corner of the article

Notes

Online First contains unedited articles in manuscript form that have been peer reviewed and accepted for publication but have not yet appeared in the paper journal (edited, typeset versions may be posted when available prior to final publication). Online First articles are citable and establish publication priority; they are indexed by PubMed from initial publication. Citations to Online First articles must include the digital object identifier (DOIs) and date of initial publication.

To order reprints of this article go to:

http://journals.bmj.com/cgi/reprintform

To subscribe to British Journal of Sports Medicine go to:

http://journals.bmj.com/subscriptions/ 


\title{
One-year follow-up of a randomised controlled trial on added splinting to eccentric exercises in chronic midportion Achilles tendinopathy
}

\author{
S de Jonge ${ }^{1}$, RJ de $\operatorname{Vos}^{2}$, HTM Van Schie ${ }^{2}$, JAN Verhaar ${ }^{2}$, A Weir ${ }^{1}, \mathrm{JL} \mathrm{Tol}^{1}$ \\ ${ }^{1}$ Sports Medicine Department. The Hague Medical Centre. Leidschendam, The Netherlands. \\ ${ }^{2}$ Department of Orthopaedics, Erasmus Medical Centre, Rotterdam. The Netherlands
}

CORRESPONDENCE TO

The Hague Medical Centre Antoniushove, Sports Medicine Department

PO Box 411, Burgemeester Banninglaan 1, 2260 AK Leidschendam, The Netherlands

E-mail: h.tol@mchaaglanden.nl

Tel: 0703574235

KEYWORDS

Achilles, tendinopathy, eccentric, night splint, PDU

ABBREVIATIONS
VISA-A - Victorian Institute of Sport Assessment - Achilles
PDU - Power Doppler Ultrasonography

WORD COUNT

Abstract: 249 words.

Manuscript: 2851 words. 


\section{Abstract}

Objective - The study examined whether the addition of a night splint to eccentric exercises is beneficial for functional outcome in chronic mid-portion Achilles tendinopathy.

Design - One-year follow-up of a randomised controlled single blinded clinical trial.

Setting - Sports medicine department in a general hospital.

Patients - 58 patients (70 tendons) were included.

Interventions - All patients completed a 12-week heavy load eccentric training programme. One group received a night splint in addition to eccentric exercises.

Main outcome measurements - Outcome scores were: Victorian Institute of Sport Assessment - Achilles (VISA-A) score, subjective patient satisfaction and neovascularisation score measured with Power Doppler Ultrasonography.

Results - For both groups the VISA-A score increased significantly (from 50 to 76 (P < 0.01 ) in the eccentric group and from 49 to 78 ( $\mathrm{P}<0.01)$ in the night splint group). No significant differences in VISA-A score were found between the groups from baseline to one year $(\mathrm{P}=0.32)$. Presence of neovessels at baseline did not predict change in VISA-A score after one year in the whole group $(\mathrm{P}=$ $0.71)$.

Conclusion - Eccentric exercises with or without a night splint improved functional outcome at one-year follow-up. At follow-up there was no significant difference in clinical outcome when a night splint was used in addition to an eccentric exercise program. Between three months and one year follow-up, a continuing increase in VISA-A score was found. Assessment of the neovascularisation score with PDU at baseline has no prognostic value on long-term clinical outcome. 


\section{Introduction}

Achilles tendinopathy is the clinical term used when the trias of a painful, swollen tendon with impaired function is present[1]. Achilles tendinopathy remains a serious injury for athletes, especially in running sports[1].

Several Scandinavian studies have reported good results with eccentric exercises in the treatment of Achilles tendinopathy[2-4]. Subsequently other studies showed only moderate results[5;6]. More recently, a systematic review concluded that treatment with eccentric exercises possibly have no benefits compared to other forms of exercises[7].

Morning stiffness is a common complaint in athletes with Achilles tendinopathy[8]. [9] hypothesised that the night splint would be effective in reducing the morning stiffness.

Functional status and return to sport activity were not reported in many previous studies. In a systematic review Kingma et al. stated that, besides pain, the functional outcome should be scored[10]. The authors proposed to use the Victorian Institute of Sports Assessment - Achilles (VISA-A) score, which measures two factors: pain and physical activity[11]. Several studies have used the VISA-A to assess outcome[6;1215].

In Achilles tendinopathy, Color - (CDU) and Power Doppler ultrasonography (PDU) shows an increased vascularity with an increased number of blood vessels, which is referred to as "neovascularisation" in the literature[16]. The pathophysiologic mechanism of these neovessels is unknown[17]. Several authors studied the presence of neovascularisation as a prognostic factor for the outcome of treatment of tendinopathy[18;19].

The first aim of this single blinded prospective randomised trial was to compare the effects at one-year follow-up of a heavy-load eccentric exercise programme compared to a heavy-load eccentric programme in combination with the use of a night splint. The second aim was to evaluate the change in neovascularisation score at one year follow-up. 


\section{Material \& Methods}

This is a follow-up study of the randomised controlled trial which initially investigated the value of additional splinting to eccentric exercises with use of the VISA-A score and PDU[5;18].

$\underline{\text { Patients }}$

Patients were referred by a general practitioner, physical therapist or a medical specialist. Volunteers without referral were also allowed for inclusion. Inclusion and exclusion criteria are summarised in table 1. The diagnosis was established based upon clinical examination. When there was pain on palpation of the Achilles tendon, 2-7 cm proximal from the distal insertion, the diagnosis of mid-portion Achilles tendinopathy was made. If there was pain on palpation of the insertion, this was considered an insertional tendinopathy and the patient was excluded.

Table 1: In and exclusion criteria

\begin{tabular}{|l|l|}
\hline Inclusion criteria & Exclusion criteria \\
\hline Age 18-70 years & Insertional disorder \\
\hline$>2$ months duration of symptoms & Tendon ruptures \\
\hline Active sport participation before onset & Systemic illness \\
\hline Wish to return to original sports level & Heavy load training in the past \\
\hline $\begin{array}{l}\text { Swollen tendon, tender on palpation and during } \\
\text { sports }\end{array}$ & Inability to perform the exercises \\
\hline Tendon thickening 2-7 cm proximal to insertion & \\
\hline
\end{tabular}

After informed consent was given all patients were included by a clinician and then examined by a single researcher. The study protocol was approved by the Medical Ethics Committee of the Hospital.

Standardised outcome measures were assessed: The VISA-A score was the primary outcome measurement to evaluate the results at follow-up. The VISA-A questionnaire was completed with minimal researcher assistance. The first question in VISA-A is related to morning stiffness (0 till 10). A higher score is associated with less duration of stiffness in the morning. Subjective patient satisfaction was scored as excellent, good, moderate or poor. PDU was performed and neovascularisation was scored according to the modified Öhberg score[18]. A detailed exercise instruction was performed. Patients were randomised into one of the treatment groups, using sealed envelopes. The researcher was blinded to this randomisation and patients were instructed not to inform the researcher. Patients who were randomised to the night splint group received further instruction from their treating physician.

Both groups performed treatment for 12 weeks. The detailed description of the treatment has been described earlier [5]. Outcome was evaluated by a blinded researcher at baseline, three months and one year. When patients needed surgery, the outcome was considered as poor patient satisfaction and the presurgical VISA-A score was obtained and the value used for the final measurement.

Ultrasound examination

On the first appointment Doppler ultrasonography was performed using a linear high frequency $8-13 \mathrm{MHz}$ transducer with a pulse repetition frequency (PRF) of $868 \mathrm{~Hz}$. (Elegra; Siemens medical systems, Erlangen, Germany). At the one-year follow-up a linear high frequency 12-15 MHz transducer with a PRF of 500-1000 Hz (MyLab30; Esaote Piemedical, Maastricht, The Netherlands) was used. Patients lay prone during the examination, with their feet hanging over the edge of the examination table. The symptomatic Achilles tendons were observed in longitudinal and transverse plane. On investigation, the researcher and the radiologists scored the neovascularisation of both Achilles tendons. This score was evaluated as 0 (no vessels visible), $1+$ (one vessel mostly in the anterior part), $2+$ (one or two vessels throughout the tendon), $3+$ (three vessels throughout the tendon) and 4+ (more than three large vessels throughout the tendon). 
Statistical analysis was performed using Statistical Package for the Social Sciences software (SPSS 12.0). To assess changes over time in the variables within groups the Wilcoxon Signed Rank test was used. The Mann Whitney U test was used to detect changes over time between the two groups. The chi-square test was used to evaluate differences in subjective patient satisfaction between the groups. Statistical significance was assumed when $P$ values were less than 0.05 . We calculated that the number of subjects to treat was 26 for each group. This sample size accounted for a $10 \%$ loss to follow-up, a significance level of 0.05 , and a power of $80 \%$. The assumptions of a delta of 10 points on the VISA-A questionnaire and a standard deviation of 12 were based on the data of previous studies[11;14]. 
Results

$\underline{\text { Patients }}$

At baseline, 70 tendons of 58 patients met the inclusion criteria. 70 tendons were randomised into two treatment groups: 34 in the eccentric group and 36 in the night splint group. The mean age was 44.6 year (26-59), the mean Body Mass Index was $25.1 \mathrm{~kg} / \mathrm{m} 2$ (20.2-34.5). The mean duration of symptoms was 30.7 months (median 44, range 2-204). There were no significant differences in patient characteristics between the groups at baseline.

50 patients (63 tendons) could be included for follow-up at one year. One patient did not answer repeated phone calls and ten patients were not able to visit our centre for PDU examination but did complete the VISA-A scores and patient satisfaction (Fig 1).

Between three months and one-year follow-up, six patients (seven tendons) did not respond to conservative treatment. All had poor/moderate patient satisfaction score at 12 weeks follow-up. These patients underwent surgical treatment after a mean duration of nine months (6-12). In these patients, the pre-surgical VISA-A scores were obtained.

\section{Eccentric training}

Some patients continued the eccentric exercises on a lower level after the 12 weeks exercise program. 11 patients (14 tendons) (22.6\%) continued for one to six months (mean 2.5 months). Eight patients (nine tendons) (14.5\%) were still doing the exercises at the one year follow-up.

\section{VISA-A score}

The VISA-A score in the eccentric group increased significantly from 50.1 at baseline to 75.7 after one year $(\mathrm{P}<0.01)$. From three months to one year the VISA-A score increased also significantly from 68.8 to 75.7 in the eccentric group $(\mathrm{P}=0.02)$. In the night splint group the VISA-A score increased from 49.2 at baseline to 78.2 at one year $(\mathrm{P}<0.01)$ (Fig 2). A significant increase was also seen from three months follow-up (65.9 points) to one-year follow-up ( $\mathrm{P}<0.01)$.

There was no significant difference found in increase in VISA-A scores between both groups from baseline to one year $(\mathrm{P}=0.36)$ and from three months to one year $(\mathrm{P}=0.24)$. (Fig 2)

The score for morning stiffness increased significantly in the eccentric group from 5.8 at baseline to 9.1 at one year follow-up $(\mathrm{P}<0.01)$. In the night splint group the score for morning stiffness increased significantly from 4.7 at baseline to 8.7 at one-year follow-up $(\mathrm{P}<0.01)$. There was no significant difference in morning stiffness between both groups at baseline $(\mathrm{P}=0.19)$, three months follow-up $(\mathrm{P}=$ $0.09)$ and one year follow-up $(\mathrm{P}=0.12)$. 
Subjective patient satisfaction

As described in table 2, patient satisfaction at one year follow-up in the eccentric group was excellent or good in $17(53.1 \%)$ tendons and moderate or poor in $15(46.9 \%)$ tendons. After one year the patient satisfaction in the night splint group was excellent or good in $21(70 \%)$ tendons and moderate or poor in nine $(30 \%)$. No significant difference in patient satisfaction was found between the eccentric group and the night splint group $(\mathrm{P}=0.20)$.

Table 2: Subjective patient satisfaction in both treatment groups at one year follow-up. Number of tendons is denoted (\%). There was no significant difference in patient satisfaction between the eccentric group and the night splint group.

\begin{tabular}{|l|l|l|}
\hline \multirow{2}{*}{} & \multicolumn{2}{|c|}{ Patient satisfaction } \\
\cline { 2 - 3 } & Moderate or poor & Excellent or good \\
\hline Eccentric group & $\mathbf{1 5 ( 4 7 \% )}$ & $\mathbf{1 7}(53 \%)$ \\
\hline Night splint group & $\mathbf{9 ( 3 0 \% )}$ & $\mathbf{2 1}(\mathbf{7 0} \%)$ \\
\hline
\end{tabular}

Ultrasonographic neovascularisation

At baseline 40 (65\%) of the 62 tendons showed some degree of neovascularisation. At one year 37 of $52(71 \%)$ showed some degree of neovascularisation. Patients with neovessels at baseline (grades 1-4) showed a mean increase of VISA-A score of 25.7 from baseline to one-year follow-up. Patients without neovessels (grade 0) at baseline showed a mean increase of 30.1 in VISA-A score. There was no significant difference in outcome measured with the VISA-A between patients with or without neovessels at baseline $(\mathrm{P}=0.71)$. (Fig 3) 


\section{$\underline{\text { Discussion }}$}

This randomised trial shows that at one year follow-up there was no difference in functional outcome between treatment with eccentric exercise only or a combination of eccentric exercises and use of a night splint in chronic Achilles tendinopathy. The improvement in VISA-A score continued after finishing the 12 weeks of therapy. Presence or absence of neovascularisation within or around the Achilles tendon could not predict outcome at long term follow-up.

All studies that compared short term versus middle and long-term outcomes, showed an improvement in the results between 6-12 weeks and one year[9;12;20]. It is questionable if the improvement after a period of three months eccentric training is caused by continuing the eccentric exercises after the prescribed three-month period. In our population almost a quarter continued the eccentric exercises for 2.5 months on average after finishing the therapy program. At one year follow-up $15 \%$ were still performing the eccentric exercises. This phenomenon has not been reported in previous studies.

Another explanation is the "slow" natural healing process of the tendon. The effect of natural healing examined with a wait and see policy, was recently studied by Rompe et al[14]. They reported that an increase of the VISA-A from 48 to 55 was found after four months without treatment. This improvement was not statistically significant. Another hypothesis is that eccentric exercises elicit longer term adaptations in extracellular matrix production. Tendons can adapt to enhanced loading conditions by changing tenocyte metabolism, leading to an alteration of structure and composition, however the process of tissue degradation, and the synthesis and incorporation of new matrix components as well as the final crosslinking of the newly produced collagen network takes time. Altered biomechanical loading conditions can influence tendon cell (tenocyte) metabolic activities, altering production and degradation of extracellular matrix components[21]. In humans it has been demonstrated using a microdialysis technique that collagen type I synthesis is increased after 12 weeks eccentric loading of initially injured human Achilles tendons[22]. From veterinary medicine we know that the tendon repair process in equine superficial digital flexors including final remodelling phase takes many months[23]. Therefore it is likely that the remodelling of the tendon tissue will continue for a longer period even after ceasing the eccentric loading regime. Ultimately this might lead to tendon structure adaptation and improved tendon function at long-term[24].

It may also be that patients might notice an initial relief and symptomatic recovery, which frequently precedes a functional recovery. It has been described that an initial symptomatic recovery reached shortly after the eccentric exercises does not automatically lead to a long-term functional recovery[25]. A postponed or extended period of restoration of tendon tissue's structure and function might be responsible for the continuing increase in VISA-A score after the eccentric exercises.

Few studies reported the functional results of more than one year follow-up. In a study in non-athletes with a mean follow-up of 15 months, Sayana and Maffulli [6] have shown VISA-A scores of 34 patients after a 12 week heavy load eccentric exercise programme. In their population they reported a significant increase of VISA-A score from 39 to 50 was found, which was a smaller improvement than in this study. A possible explanation is the difference in study population. In the study of Sayana and Maffulli the patients were non-athletes, whereas in this study the included patients were all participating in sports. It could be postulated that active individuals do have a better compliance for the prescribed exercises. The VISA-A scores after one year in the study of Brown et al [12] are more comparable with the present study. These authors showed an improvement from 62 to 95 in 18 patients treated with eccentric exercises plus an injection of aprotinin or placebo. Other studies showed also an increase of VISA-A after finishing the eccentric programme[5;14;15].

In contrast to the hypothesis that a night splint would reduce morning stiffness, the results in the present study did not show a significant benefit of using a night splint for the duration of stiffness in the morning.

There is conflicting evidence on the significance of neovascularisation in Achilles tendinopathy[26]. Peers et al. [27] found a correlation between neovascularisation and pain score and Reiter et al. [28] reported a relationship between presence of neovascularisation and a worse VISA-A score. However, these data were not supported by Zanetti et al. [19] and de Vos et al [18] found no relationship 
between the Visual Analogue Scale (VAS) score or VISA-A score and the presence of neovascularisation.

In this study there was no relationship between neovascularisation at baseline and clinical outcome at one year. Zanetti et al. [19] and de Vos et al. [18] analysed the presence of neovessels at baseline related to outcome after three months of conservative treatment and reported similar results.

The use of a night splint in Achilles tendinopathy has been studied by Roos et al [9]. They reported a pain reduction of 35-42\% at one year follow-up in all patients treated with eccentric exercises, a night splint or a combination of both. There was no significant difference in pain reduction between these groups after one year.

At one-year follow-up another PDU device was used. Although the pulse repetition frequency did not differ much, comparison between baseline and one-year follow-up could be unreliable. Another weakness of this study is the high number of patients who were not able to attend for PDU examination at one-year follow-up. An intention-to-treat analysis was used for the patients who received conservative treatment to avoid bias. However, because some patients received surgical treatment they were not comparable to the group of patients treated conservatively. Therefore, these tendons were scored as poor patient satisfaction with the pre-surgical VISA-A score. A type II error may have occurred in the present study, however with a total of 62 patients analysed at follow-up a high power was achieved. 


\section{Conclusion}

Eccentric exercises, with or without a night splint, improved functional outcome after one-year followup in patients with chronic Achilles tendinopathy. At follow-up there was no significant difference when a night splint was used in addition to an eccentric exercise program.

There was a continued improvement in functional outcome between three months and one year. $37 \%$ of the patients treated with eccentric exercises continued to perform them after the three months prescribed duration.

Presence of neovascularisation at baseline did not predict pain and functional outcome at one-year follow-up. $71 \%$ of the tendons still showed some degree of neovascularisation at follow-up. 


\section{ACKNOWLEDGEMENTS}

We would like to thank ms. M. de Mos (PhD student, Orthopaedic Research Laboratory, Erasmus Medical Centre Rotterdam) for her contribution in the field of basic biological response to exercise.

\section{LICENCE}

The Corresponding Author has the right to grant on behalf of all authors and does grant on behalf of all authors, an exclusive licence (or non exclusive for government employees) on a worldwide basis to the BMJ Publishing Group Ltd and its Licensees to permit this article (if accepted) to be published in Journal (British Journal of Sports Medicine) editions and any other BMJPGL products to exploit all subsidiary rights, as set out in our licence (http://bjsm.bmjjournals.com/misc/ifora/licenceform.shtml).

\section{COMPETING INTERESTS}

None

WHAT IS ALREADY KNOWN ON THIS TOPIC?

Eccentric exercises is widely used and is effective in pain reduction in the treatment of chronic Achilles tendinopathy.

Role of neovascularisation as a prognostic factor remains questionable.

\section{WHAT THIS STUDY ADDS?}

There is no benefit of adding a night splint to eccentric exercises in treatment of Achilles tendinopathy.

There is a continuing improvement in pain and function between three months and one year follow-up of conservative treatment.

Neovascularisation score at baseline has no prognostic value for clinical outcome. 


\section{Reference List}

1 Maffulli N, Wong J, Almekinders LC. Types and epidemiology of tendinopathy. Clin Sports Med 2003;22:675-92.

2 Alfredson H, Pietila T, Jonsson P, Lorentzon R. Heavy-load eccentric calf muscle training for the treatment of chronic Achilles tendinosis. Am J Sports Med 1998;26:360-6.

3 Fahlstrom M, Jonsson P, Lorentzon R, Alfredson H. Chronic Achilles tendon pain treated with eccentric calf-muscle training. Knee Surg Sports Traumatol Arthrosc 2003;11:327-33.

4 Mafi N, Lorentzon R, Alfredson H. Superior short-term results with eccentric calf muscle training compared to concentric training in a randomized prospective multicenter study on patients with chronic Achilles tendinosis. Knee Surg Sports Traumatol Arthrosc 2001;9:42-7.

5 de Vos RJ, Weir A, Visser RJ, de Winter T, Tol JL. The additional value of a night splint to eccentric exercises in chronic midportion Achilles tendinopathy: a randomised controlled trial. Br J Sports Med 2007;41:e5.

6 Sayana MK, Maffulli N. Eccentric calf muscle training in non-athletic patients with Achilles tendinopathy. J Sci Med Sport 2007;10:52-8.

7 Wasielewski NJ, Kotsko KM. Does eccentric exercise reduce pain and improve strength in physically active adults with symptomatic lower extremity tendinosis? A systematic review. $J$ Athl Train 2007;42:409-21.

8 Alfredson H, Cook JL. Pain in the Achilles Region. In: Brukner P, Khan KM, editors. Clinical Sports Medicine. 3 ed. 2006. p. 590-611.

9 Roos EM, Engstrom M, Lagerquist A, Soderberg B. Clinical improvement after 6 weeks of eccentric exercise in patients with mid-portion Achilles tendinopathy -- a randomized trial with 1-year follow-up. Scand J Med Sci Sports 2004;14:286-95.

10 Kingma JJ, de Knikker R, Wittink HM, Takken T. Eccentric overload training in patients with chronic Achilles tendinopathy: a systematic review. Br J Sports Med 2007;41:e3.

11 Robinson JM, Cook JL, Purdam C, Visentini PJ, Ross J, Maffulli N, et al. The VISA-A questionnaire: a valid and reliable index of the clinical severity of Achilles tendinopathy. $\mathrm{Br} J$ Sports Med 2001;35:335-41.

12 Brown R, Orchard J, Kinchington M, Hooper A, Nalder G. Aprotinin in the management of Achilles tendinopathy: a randomised controlled trial. Br J Sports Med 2006;40:275-9.

13 Paoloni JA, Murrell GA. Three-year followup study of topical glyceryl trinitrate treatment of chronic noninsertional Achilles tendinopathy. Foot Ankle Int 2007;28:1064-8.

14 Rompe JD, Furia J, Maffulli N. Eccentric loading compared with shock wave treatment for chronic insertional achilles tendinopathy. A randomized, controlled trial. J Bone Joint Surg Am 2008;90:52-61.

15 Tumilty S, Munn J, Abbott JH, McDonough S, Hurley DA, Baxter GD. Laser therapy in the treatment of achilles tendinopathy: a pilot study. Photomed Laser Surg 2008;26:25-30. 
17 Alfredson H. Chronic midportion Achilles tendinopathy: an update on research and treatment. Clin Sports Med 2003;22:727-41.

18 de Vos RJ, Weir A, Cobben LP, Tol JL. The value of power Doppler ultrasonography in Achilles tendinopathy: a prospective study. Am J Sports Med 2007;35:1696-701.

19 Zanetti M, Metzdorf A, Kundert HP, Zollinger H, Vienne P, Seifert B, et al. Achilles tendons: clinical relevance of neovascularization diagnosed with power Doppler US. Radiology 2003;227:556-60.

20 Norregaard J, Larsen CC, Bieler T, Langberg H. Eccentric exercise in treatment of Achilles tendinopathy. Scand J Med Sci Sports 2007;17:133-8.

21 Kjaer M, Magnusson P, Krogsgaard M, Boysen MJ, Olesen J, Heinemeier K, et al. Extracellular matrix adaptation of tendon and skeletal muscle to exercise. J Anat 2006;208:445-50.

22 Langberg H, Ellingsgaard H, Madsen T, Jansson J, Magnusson SP, Aagaard P, et al. Eccentric rehabilitation exercise increases peritendinous type I collagen synthesis in humans with Achilles tendinosis. Scand J Med Sci Sports 2007;17:61-6.

23 van Schie HT. Ultrasonographic Tissue Characterization of equine superficial digital flexor tendons; development and applications of computer-aided image analysis. Utrecht, The Netherlands: 2004.

24 Wang JH. Mechanobiology of tendon. J Biomech 2006;39:1563-82.

25 Silbernagel KG, Thomee R, Thomee P, Karlsson J. Eccentric overload training for patients with chronic Achilles tendon pain--a randomised controlled study with reliability testing of the evaluation methods. Scand J Med Sci Sports 2001;11:197-206.

26 Knobloch K. The use of a neovascularization score to predict clinical severity in Achilles tendinopathy. Am J Sports Med 2008;36:395-7.

27 Peers KH, Brys PP, Lysens RJ. Correlation between power Doppler ultrasonography and clinical severity in Achilles tendinopathy. Int Orthop 2003;27:180-3.

28 Reiter M, Ulreich N, Dirisamer A, Tscholakoff D, Bucek RA. Colour and power Doppler sonography in symptomatic Achilles tendon disease. Int J Sports Med 2004;25:301-5. 


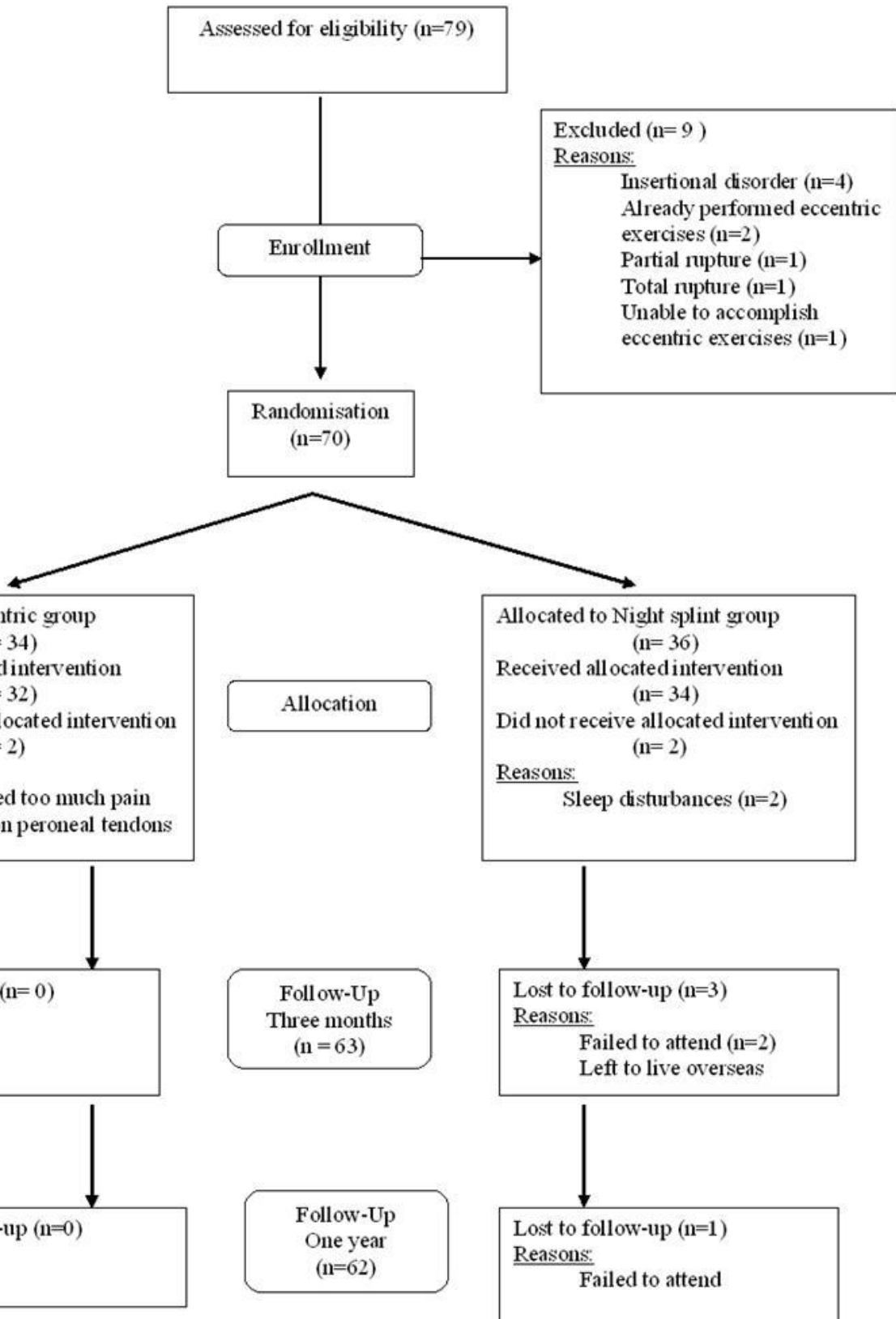


Figure 2: Changes in VISA-A in both treatment groups at baseline, three months follow-up and one year follow-up. No significant differences were found between the two treatment groups. A significant increase for the eccentric and night splint group between three months and one year follow-up was found.

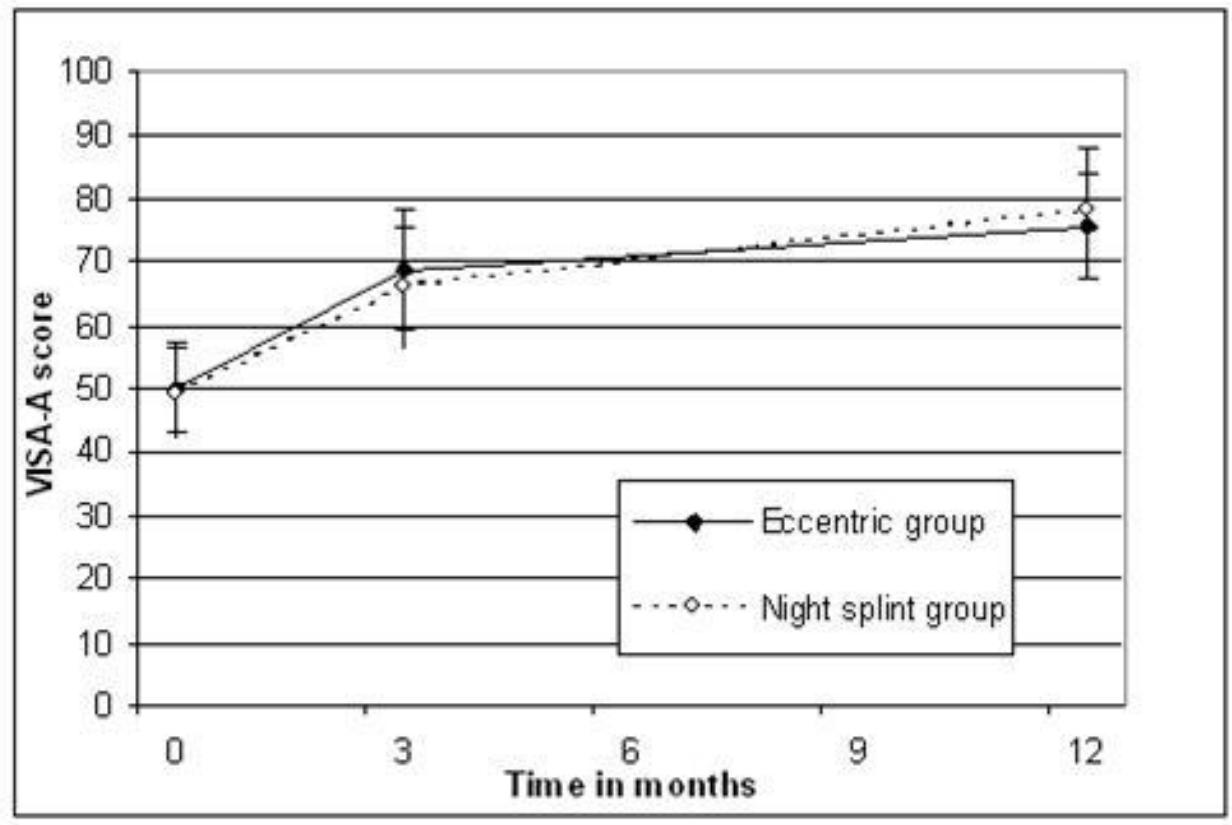


Figure 3: Changes in VISA-A at baseline, three months and one year follow-up in patients with neovessels (grades 1-4) at baseline and without neovessels at baseline (grade 0). There was no significant difference in improvement of the VISA-A score between these groups at three months and one year follow-up.

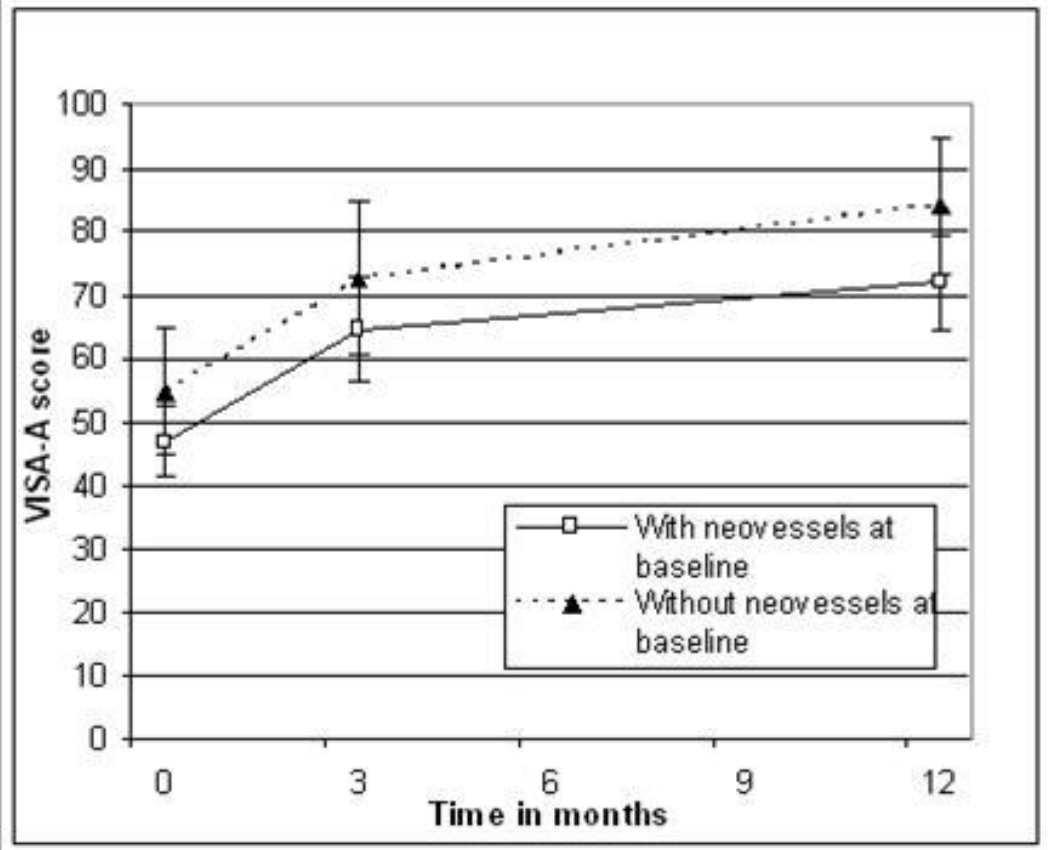

